\title{
A New Simple Chaotic Lorenz-Type System and Its Digital Realization Using a TFT Touch-Screen Display Embedded System
}

\author{
Rodrigo Méndez-Ramírez, ${ }^{1}$ Adrian Arellano-Delgado, ${ }^{2}$ \\ César Cruz-Hernández, ${ }^{1}$ and Rigoberto Martínez-Clark ${ }^{1}$ \\ ${ }^{1}$ Electronics and Telecommunications Department, Scientific Research and Advanced Studies Center of Ensenada, \\ Ensenada, BC, Mexico \\ ${ }^{2}$ CONACYT-Autonomous Baja California University (UABC), Ensenada, BC, Mexico
}

Correspondence should be addressed to César Cruz-Hernández; ccruz@cicese.mx

Received 14 February 2017; Revised 5 May 2017; Accepted 31 May 2017; Published 26 July 2017

Academic Editor: Giacomo Innocenti

Copyright ( 2017 Rodrigo Méndez-Ramírez et al. This is an open access article distributed under the Creative Commons Attribution License, which permits unrestricted use, distribution, and reproduction in any medium, provided the original work is properly cited.

\begin{abstract}
This paper presents a new three-dimensional autonomous chaotic system. The proposed system generates a chaotic attractor with the variation of two parameters. Analytical and numerical studies of the dynamic properties to generate chaos, for continuous version (CV) and discretized version (DV), for the new chaotic system (NCS) were conducted. The CV of the NCS was implemented by using an electronic circuit with operational amplifiers (OAs). In addition, the presence of chaos for DV of the NCS was proved by using the analytical and numerical degradation tests; the time series was calculated to determine the behavior of Lyapunov exponents (LEs). Finally, the DV of NCS was implemented, in real-time, by using a novel embedded system (ES) Mikromedia Plus for PIC32MX7 that includes one microcontroller PIC32 and one thin film transistor touch-screen display (TFTTSD), together with external digital-to-analog converters (DACs).
\end{abstract}

\section{Introduction}

In recent years, chaotic systems have attracted the attention of the scientific community due to their potential applications in several areas of science and engineering, as an interesting nonlinear phenomenon with different applications in biology, secure communication, complex networks, experimental network synchronization, fingerprint encryption, among others [1-15]. In 1963, Lorenz proposed a three-dimensional system of two scrolls; this is recognized as the first reported chaotic model [16]. Since then, many chaotic systems like Lorenz-and their chaotic behavior-have been reported in the literature, for example, [17-23]. Currently, we can mention some new chaotic systems reported in the literature [24-33].

The chaotic systems usually are implemented by using electronic circuits in continuous (CV) and discretized versions (DV). The CVs usually are represented by using OAs
$[31,34]$. For DVs, Matlab or Labview allows simulating the dynamical behaviors of discretized chaotic systems to desirably obtain the less degradation with respect to CVs, and their implementations are reproduced by using ESs as FPGAs [35], DSPs [36], or microcontrollers [37-39].

In this paper, we propose a new simple chaotic system, which is derived from the Lorenz system [16]. The novelty of the proposed chaotic system is the combination of different characteristics that it presents: two critical parameters, only two nonlinearities, low complexity time, low iterations per second, and a larger step size for the discretized version where chaos is preserved, low-cost electronic implementation; also it is flexible and robust with respect to some recent attractors reported in the literature; see, for example, $[22,23]$. As a consequence, all these features result in a high ease of implementation that may be of great interest in engineering applications, for example, in cryptography, biometric 
systems, telemedicine, and secure communications; see, for example, $[10,12,13,37]$. To the best of our knowledge the electronical implementation in a portable TFTTSD device of DV of chaotic systems, for the reproduction of their nonlinear dynamics in real-time, is new.

The paper is organized as follows: Section 2 reports basic analytical proof and extensive numerical tests to verify the existence of chaos in the proposed NCS. Section 3 presents two electronic implementations to reproduce the NCS: the first by means of OAs and the second of a novel proposed ES by using one TFTTSD and external DACs. In addition, a degradation study taking into account the preservation of chaos is conducted; a comparison among the NCS and some other chaotic systems reported in the literature is made. In Section 4, we give a complete description of digital implementation process of the DV of NSC with the corresponding robustness diagram to determine regions in which the existence of chaos is guaranteed. Finally, in Section 5 we draw some concluding remarks.

\section{Basic Analysis and Characterization of NCS}

This section presents the state equations of NCS and some numerical and analytical tests to verify the chaos existence in the proposed system. The NCS is built starting from inspecting and modification of Lorenz system [16]. The proposed NCS is described by

$$
\begin{aligned}
& \dot{x}=-a x-b y z, \\
& \dot{y}=-x+c y, \\
& \dot{z}=d-y^{2}-z .
\end{aligned}
$$

The proposed system has seven terms, two quadratic nonlinearities, and four parameters $a, b, c, d \in \mathbb{R}^{+}$, where $b$ and $d$ are characterized as the bifurcation parameters. The nonlinear NCS (1) is chaotic with $a=2, b=2, c=0.5$, and $d=4$.

System (1) is symmetrical about the $z$-axis due to its invariance under the coordinate transformation $(x, y, z) \rightarrow$ $(-x,-y, z)$. The symmetry is not associated with the $a, b, c$, and $d$ parameters.

The divergence for a 3-dimensional flow of dynamical system is defined by

$$
\nabla V=\frac{\partial \dot{x}}{\partial x}+\frac{\partial \dot{y}}{\partial y}+\frac{\partial \dot{z}}{\partial z}=-a+c-1=-2.5<0 .
$$

Therefore, the above analysis proves that our system is dissipative. The exponential contraction rate is calculated as follows:

$$
\frac{d V}{d t}=(\nabla V) V \longrightarrow V=V_{0} e^{-2.5 t}
$$

where each volume containing the system trajectory shrinks to zero as $t \rightarrow \infty$ at an exponential rate of $-2.5 t$. System orbits are ultimately confined into a specific limit set of zero volume, and the asymptotic motion settles onto an attractor. Thereby, the existence on attractor is proved.
The boundness of the chaotic trajectories of system (1) is proved by means of the following theorem. The boundness of a NCS by using similar approach was reported in $[40,41]$.

Theorem 1. Suppose that the parameters $a, b, c$, and $d$ of system (1) are positive. Then, the orbits of system (1) including chaotic orbits are confined in a bounded region.

Proof. Consider the candidate Lyapunov function

$$
V(x, y, z)=\frac{1}{2}\left(x^{2}+y^{2}+z^{2}\right)
$$

and the time derivative of $V(x, y, z)$ along the trajectories of the NCS (1) is given by

$$
\begin{aligned}
\dot{V}(x, y, z)= & x \dot{x}+y \dot{y}+z \dot{z} \\
\dot{V}(x, y, z)= & -a x^{2}-b x y z+c y^{2}-x y+d z-z y^{2}-z^{2} \\
= & -\left(\frac{b y z}{2 \sqrt{a}}+\sqrt{a} x\right)^{2}+\left(\frac{x}{2 \sqrt{c}}-\sqrt{c} y\right)^{2} \\
& -\frac{x^{2}}{4 c}-\left(z-\frac{d}{2}\right)^{2}+\frac{d^{2}}{4} \\
& +\left(\frac{b y z}{2 \sqrt{a}}-\frac{\sqrt{a} y}{b}\right)^{2}-\frac{a y^{2}}{b^{2}} .
\end{aligned}
$$

Let $R_{0}$ be the sufficiently large region so that for all trajectories $(x, y, z)$ satisfy $V(x, y, z)=R$ for $R>R_{0}$ with the condition

$$
\begin{aligned}
& \left(\frac{b y z}{2 \sqrt{a}}+\sqrt{a} x\right)^{2}+\left(z-\frac{d}{2}\right)^{2}+\frac{a y^{2}}{b^{2}}+\frac{x^{2}}{4 c} \\
& >\left(\frac{x}{2 \sqrt{c}}-\sqrt{c} y\right)^{2}+\left(\frac{b y z}{2 \sqrt{a}}-\frac{\sqrt{a} y}{b}\right)^{2}+\frac{d^{2}}{4} .
\end{aligned}
$$

Consequently on the surface $\{(x, y, z) / V(x, y, z)\}=R$. Since $R>R_{0}$ we can write $\dot{V}(x, y, z)<0$, or the set $\{(x, y, z) / V(x, y, z)\} \leq R$ is a confined region for all the trajectories of chaotic system (1).

The number of the equilibrium points and their stabilities determine the behavior of system (1); it can be found by setting $\dot{x}=\dot{y}=\dot{z}=0$ and $a, b, c, d>0$. The proposed system (1) has five fixed points: $P_{0}(0,0,0), P_{1}, P_{2}, P_{3}$, $P_{4}( \pm c \sqrt{d+a c / b}, \pm \sqrt{d+a c / b},-a c / b)$. The Jacobean matrix of CV-system (1) is given by

$$
J_{\mathrm{CV}}=\left(\begin{array}{ccc}
-a & -b z & -b y \\
-1 & c & 0 \\
0 & -2 y & -1
\end{array}\right),
$$

and the characteristic polynomial of (7) is as follows:

$$
\begin{aligned}
\operatorname{det}(\lambda I-J)= & \lambda^{3}+(a-c+1) \lambda^{2}+(a-c-a c-b z) \lambda \\
& +2 b y^{2}-a c-b z=0 .
\end{aligned}
$$


TABLE 1: Stability analysis equilibrium points for NCS.

\begin{tabular}{lcc}
\hline Point & Eigenvalues & Stability \\
\hline & $\lambda_{1}=-2$ & \\
$P_{2}=-1$ & $\lambda_{1}, \lambda_{2}<0$, and $\lambda_{3}>0$, unstable saddle point \\
& $\lambda_{3}=0.5$ & \\
\hline & $\lambda_{1}=-3.52387$ & \\
$P_{1}, P_{2}, P_{3}, P_{4}$ & $\lambda_{2}=0.51193+2.20134 i$ & $\lambda_{1}<0$ and the real part of $\lambda_{2}, \lambda_{3}>0$, unstable saddle points \\
& $\lambda_{3}=0.51193+2.20134 i$ & \\
\hline
\end{tabular}

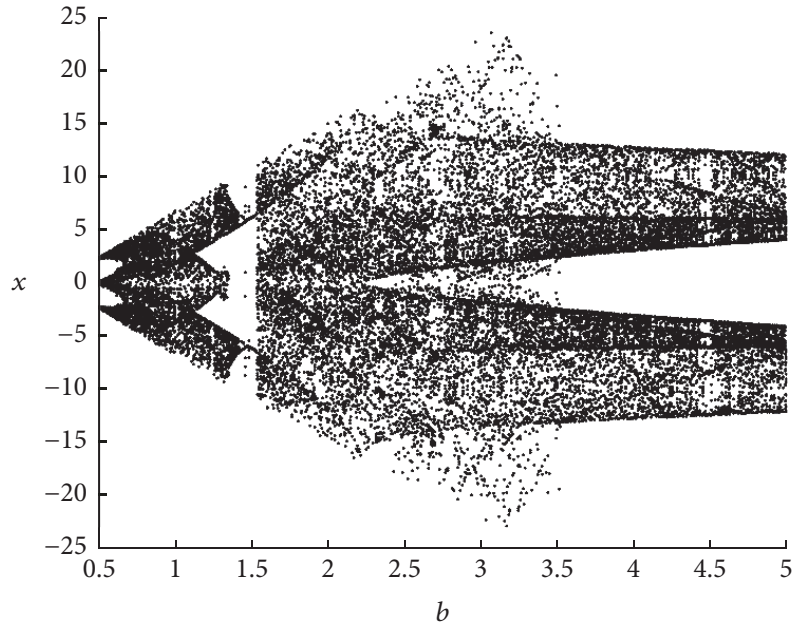

(a)

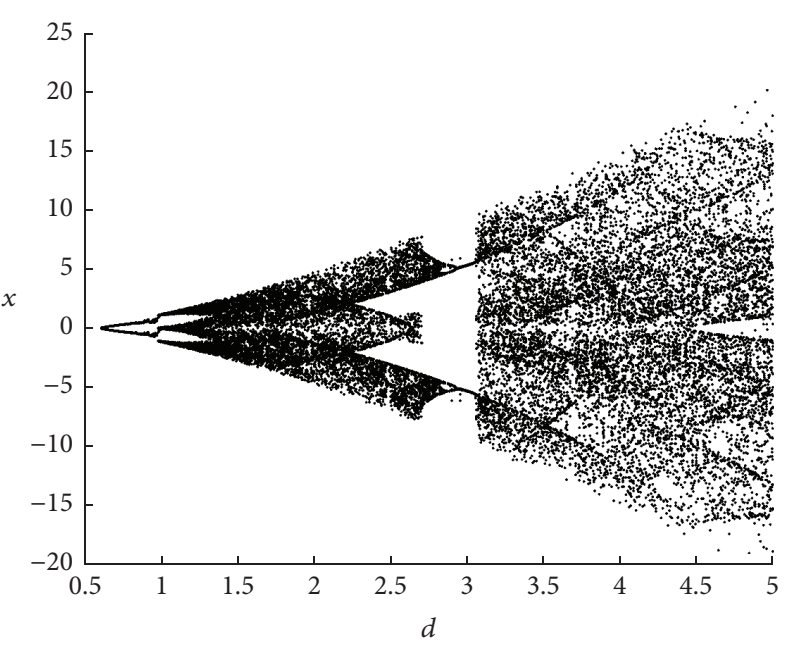

(b)

Figure 1: Bifurcation diagrams of parameters $b$ and $d$, versus state $x$ using the initial conditions $x_{0}=y_{0}=z_{0}=1$ of system (1): (a) variations of $b=[0.5,5]$ and (b) variations of $d=[0.5,5]$.

Evaluating with parameters $a=2, b=2, c=0.5$, and $d=$ 4 in (8), the stability in the equilibrium points $P_{0,1,2,3,4}$ was studied. Table 1 shows the stability results of equilibria where all points of NCS are saddle-focus unstable nodes.

The bifurcation diagram is built to visualize the transitions between periodic and chaotic motions of the proposed system, with the variation of the critical parameter $b$ or $d$ of NCS (1); for more details of the numerical algorithm to obtain the bifurcation diagram see [42]. Figure 1(a) shows the bifurcation diagram of system (1), where the parameters $a=2, c=2$, and $d=4$ were fixed, and $b$ is varied. In addition, Figure 1(b) shows the bifurcation diagram of system (1) where the parameters $a=2, b=2$, and $c=0.5$ were fixed, and $d$ is varied. From Figure 1 we conclude that system (1) is also robust because it has a large chaotic behavior for parameters $b$ and $d$ to guarantee chaotic behaviors: fixed point, limit cycle, and strange attractor.

In this numerical study, the parameter $b$ is fixed and the parameter $d$ is choice as bifurcation parameter. The initial conditions $x_{0}=y_{0}=z_{0}=1$ and parameters $a=2, b=2$, $c=0.5$, and $d=4$ were chosen for all numerical and experimental tests.

To prove the presence of chaos on the NCS (1), the LEs are calculated using the method reported in $[43,44]$. Figure 2 shows the results of the LEs where 10000 time units were considered in the analysis. Figure 2(a) shows the evolution of LEs where the obtained results are $L_{1}=0.24914, L_{2}=0$, and $L_{3}=-2.7497$. Figure 2 (b) shows evolution of LEs considering the variation of bifurcation parameter $d=[0.5,5]$.

The fractal dimension, commonly known as KaplanYorke dimension $D_{\mathrm{KY}}$, of this system is

$$
D_{\mathrm{KY}}=j+\frac{1}{\left|L_{j+1}\right|} \sum_{i=1}^{j} L_{i}=2+\frac{L_{1}+L_{2}}{\left|L_{3}\right|}=2.0908 .
$$

The NCS exhibits complex and abundant dynamics behaviors; see Figure 3 where chaotic attractors are shown.

\section{Electronic Implementations}

This section presents two electronic implementations for system (1): (i) the CV was simulated and implemented with the design of one circuit by using OAs, and (ii) the DV was implemented with design of an ES where one degradation study of NCS is also given.

3.1. Electronic Circuit Design for CV of NCS. For the electronic implementation of CV-system (1) the attenuation factor of 20 for each of the state variables $x=20 u, y=20 v$, and $z=20 w$ 


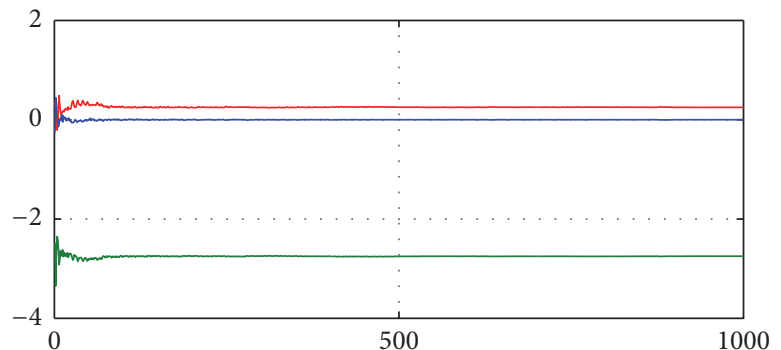

$\begin{aligned}- & L_{1} \\ - & L_{2} \\ - & L_{3}\end{aligned}$

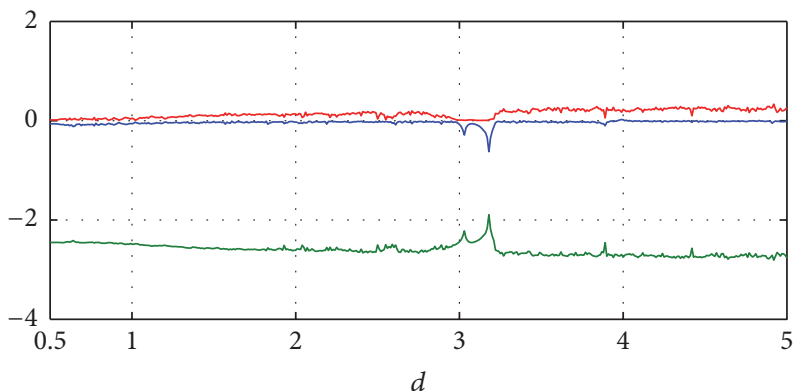

$-L_{1}$
$-L_{2}$
$-L_{3}$

(a)

(b)

FIGURE 2: LEs of system (1): (a) the bifurcation parameter fixed in $d=4$ and (b) variation of bifurcation parameter $d=[0.5,5]$.

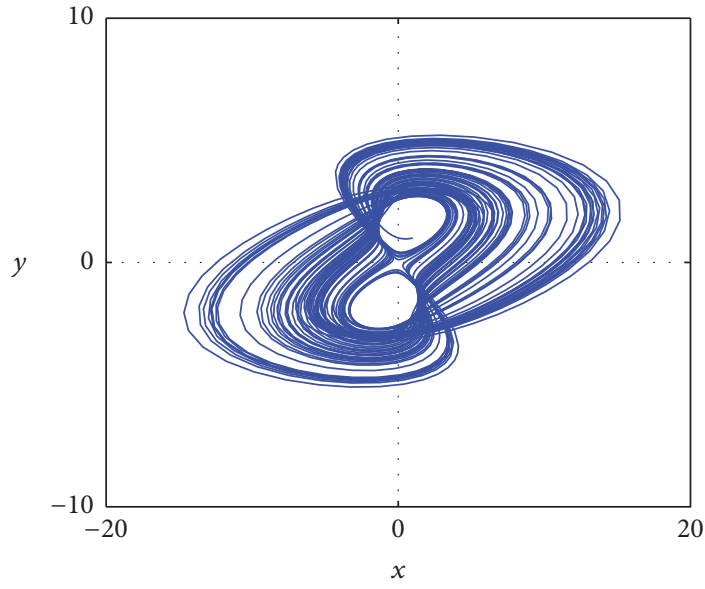

(a)

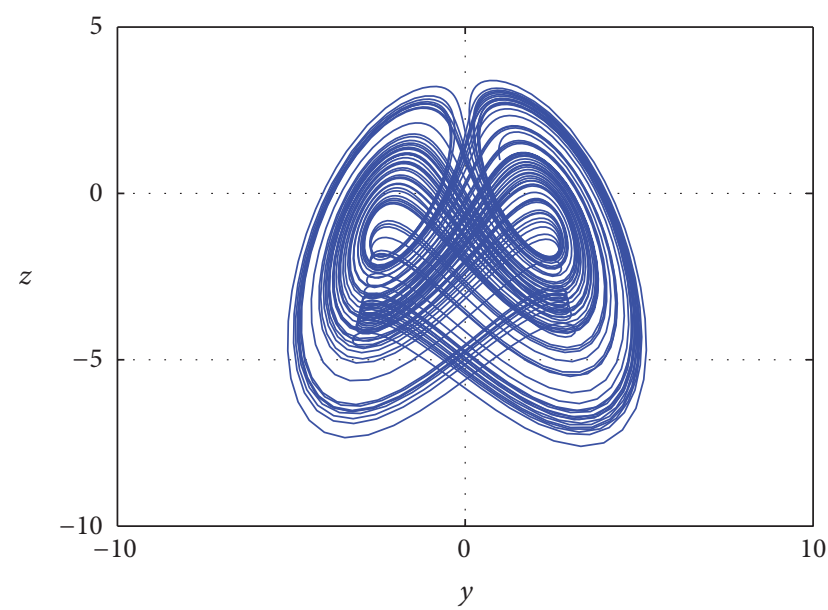

(c)

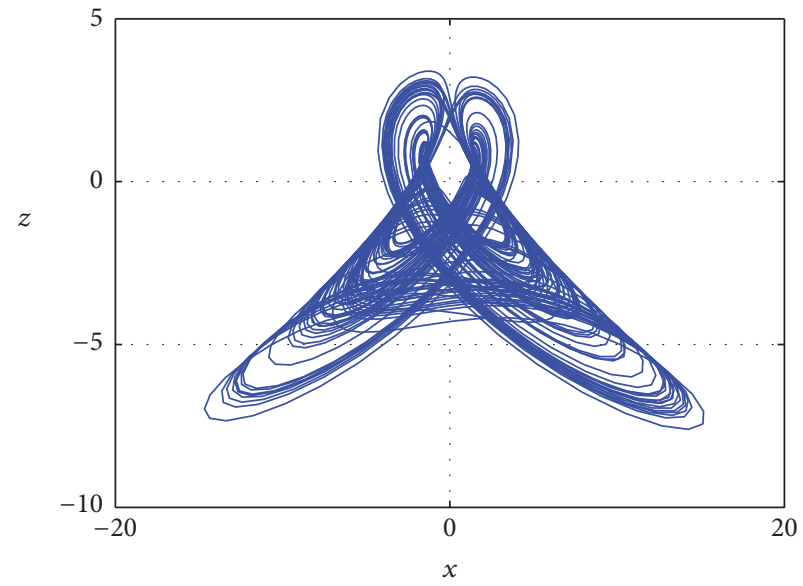

(b)

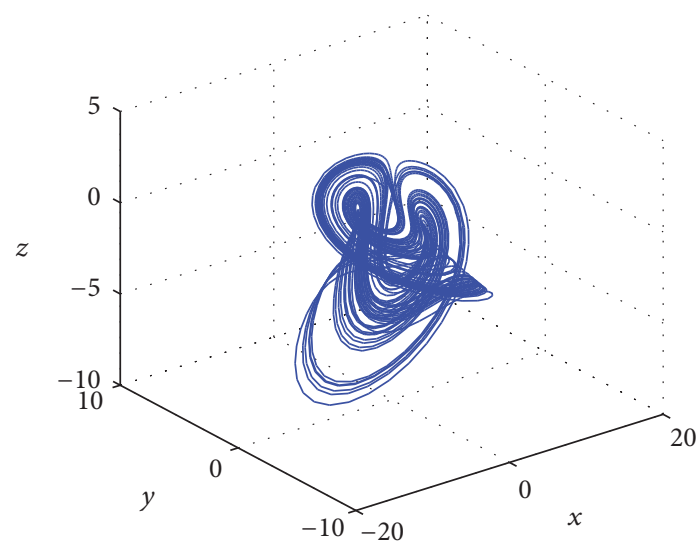

(d)

Figure 3: Chaotic attractors of system (1): (a) phase plane $x$ versus $y$, (b) phase plane $x$ versus $z$, (c) phase plane $y$ versus $z$, and (d) phase space $x$ versus $y$ versus $z$. 

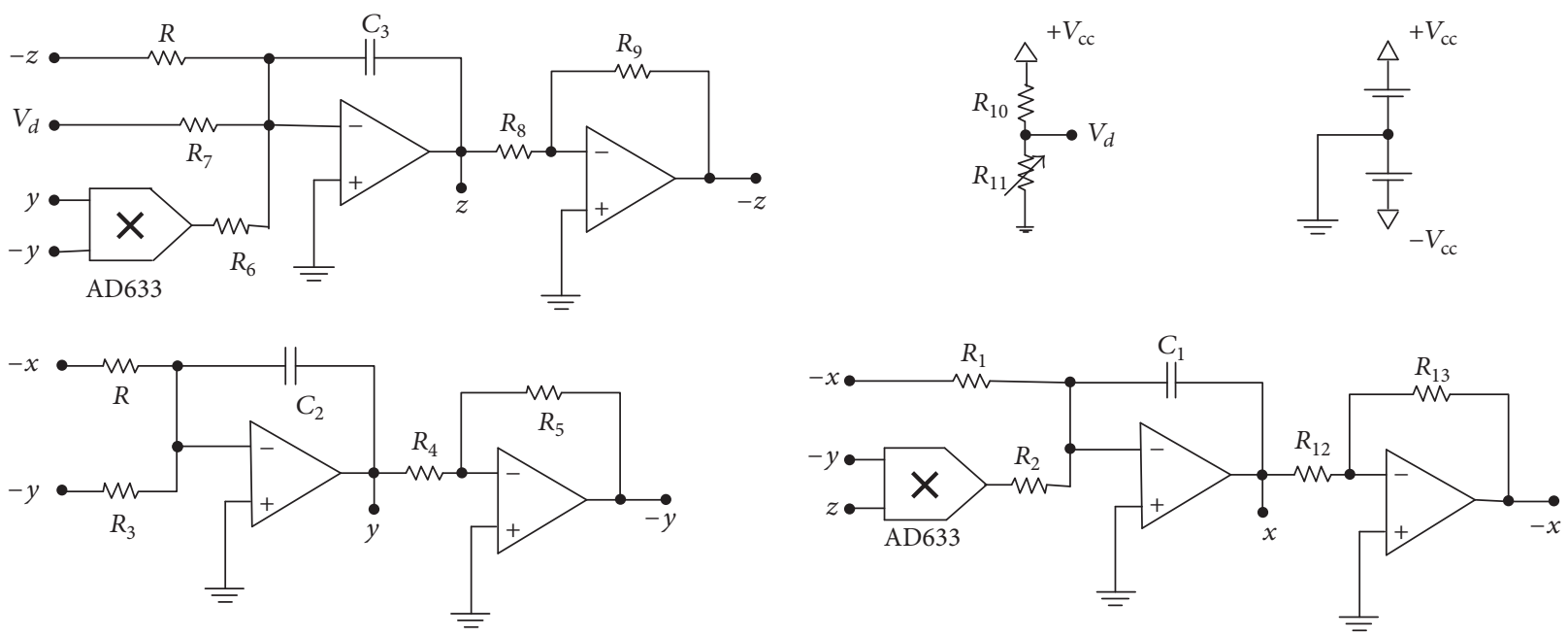

FIGURE 4: Schematic diagram of the equivalent circuit of system (11).

was calculated. Replacing the new variables on system (1), we obtain the following system:

$$
\begin{aligned}
& \dot{u}=-2 u-40 v w, \\
& \dot{v}=-u+0.5 v, \\
& \dot{w}=0.2-20 v^{2}-w .
\end{aligned}
$$

Replacing the state variables $x=u, y=v$, and $z=w$ in system (10), the representation of circuit is

$$
\begin{aligned}
& \dot{x}=\frac{1}{R C_{l}}\left(-\frac{R}{R_{l}} x-\frac{R}{10 R_{l 0}} y z\right), \\
& \dot{y}=\frac{1}{R C_{2}}\left(-x+\frac{R}{R_{5}} y\right), \\
& \dot{z}=\frac{1}{R C_{3}}\left(\frac{R}{R_{9}} b-\frac{R}{10 R_{8}} y^{2}-z\right),
\end{aligned}
$$

where the components are OAs TL084, multipliers AD633, $C_{1}=C_{2}=C_{3}=100 \mathrm{pF}, R_{1}=500 \mathrm{k} \Omega, R_{2}=47 \mathrm{k} \Omega, R=$ $R_{10}=1 \mathrm{M} \Omega, R_{3}=2 \mathrm{M} \Omega, R_{6}=100 \mathrm{k} \Omega, R_{4}=R_{5}=R_{8}=$ $R_{9}=R_{12}=R_{13}=10 \mathrm{k} \Omega$, and $R_{7}=5 \mathrm{M} \Omega$, the bifurcation parameter is fixed in $d=4$ with $R_{11}=287 \mathrm{k} \Omega$, and the circuit of Figure 4 is powered with $+V_{c c}=18 \mathrm{~V}$ and $-V_{c c}=-18 \mathrm{~V}$. To see a change in the dynamical behavior of system (11), it is recommend to represent the bifurcation parameter $d$ with a variable resistor of $R_{11(\mathrm{VAR})}=1 \mathrm{M} \Omega$; this voltage was referred to as $V_{d}$. Figure 4 shows the equivalent circuit of system (11).

In order to compare the experimental with numerical results, Figure 5 shows the comparison on phase planes of system (11) between Multisim simulation and electronic circuit implementation; we can see that the corresponding attractors are similar with respect to those shown in Figure 3.

3.2. DV of NCS and Its Digital Implementation. It is well known that Euler's method in order to discretize a continuous system is derived from the expansion of Taylor's series, when the quadratic and upper order term are truncated. The Euler method to approximate the ordinary differential equations (ODEs),

$$
\begin{aligned}
\dot{\mathbf{x}} & =\mathbf{f}(\mathbf{x}), \\
\mathbf{x}(0) & =\mathbf{x}_{0}, \\
\mathbf{x} & \in \mathbb{R}^{N},
\end{aligned}
$$

is given by

$$
\mathbf{x}_{(n+1)}=\mathbf{x}_{(n)}+\tau \mathbf{f}\left(\mathbf{x}_{(n)}\right),
$$

where $\tau$ is the step size and $n$ is the iteration number that represent the time in discrete version. Euler's discretization (13) was considered to obtain the DV of the proposed NCS (1) as follows:

$$
\begin{aligned}
& x_{(n+1)}=x_{(n)}+\tau\left(-a x_{(n)}-b y_{(n)} z_{(n)}\right), \\
& y_{(n+1)}=y_{(n)}+\tau\left(-x_{(n)}+c y_{(n)}\right), \\
& z_{(n+1)}=z_{(n)}+\tau\left(d-y_{(n)}^{2}-z_{(n)}\right) .
\end{aligned}
$$

The advantage of Euler's method is that it is easy to understand and simple to execute as numerical algorithm; in addition it has low time complexity. Even though its low accuracy, this method is widely used for solving (numerically) ODEs; for more details, please see [45].

The Matlab simulations of the DV-system (14) were carried out by using $\tau=0.005$ and $n=40000$. Figure 6 shows the phase space $x_{(n)}$ versus $y_{(n)}$ versus $z_{(n)}$ of DV-system (14).

Microchip Technology Inc. is the manufacturer of microcontroller PIC32, their numerical results were represented in floating points 32 bits according to the IEEE-754 Compliant Floating Point Routines [46]. The standard IEEE-754 also is included in Matlab for 32-bit version [45]. The microcontroller PIC32 was programmed by using Mikroc Pro for Pic 32 compiler that includes the standard IEEE-754; this means 


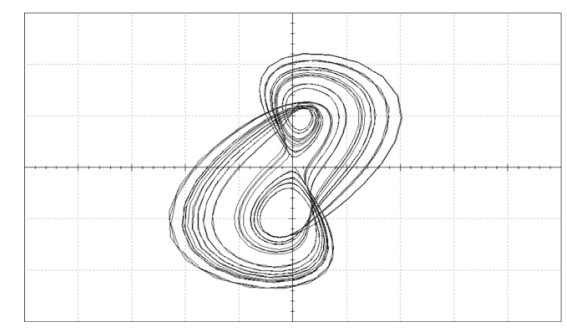

(a)

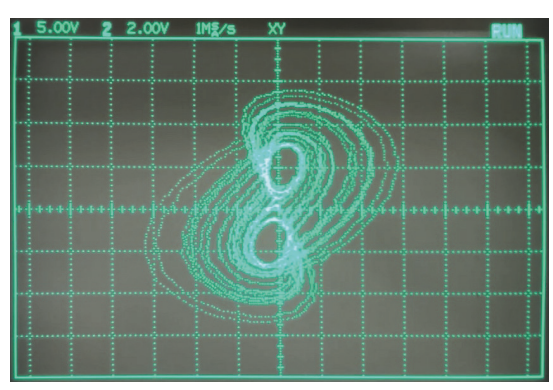

(d)

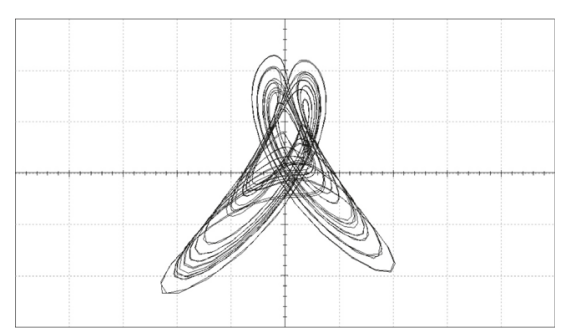

(b)

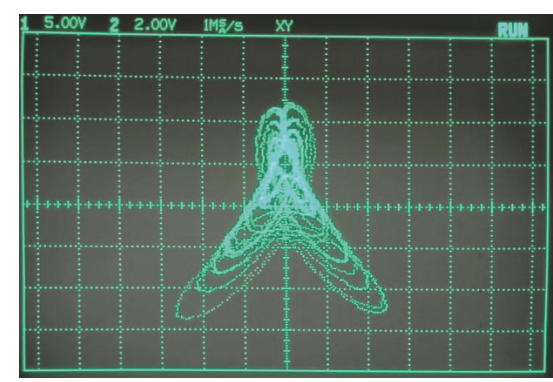

(e)

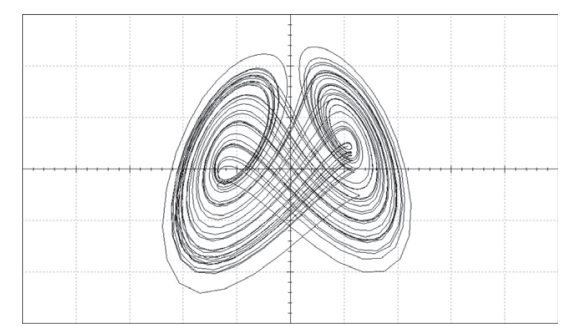

(c)

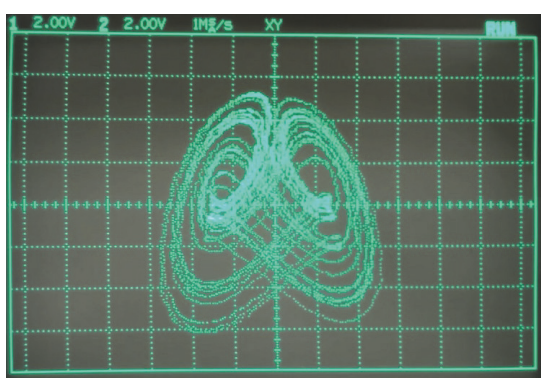

(f)

Figure 5: Comparison on phase planes between simulation and circuit implementation of system (11). Multisim simulation: (a) $x$ versus $y$, (b) $x$ versus $z$, and (c) $y$ versus $z$, and electronic circuit implementation: (d) $x$ versus $y$, (e) $x$ versus $z$, and (f) $y$ versus $z$.

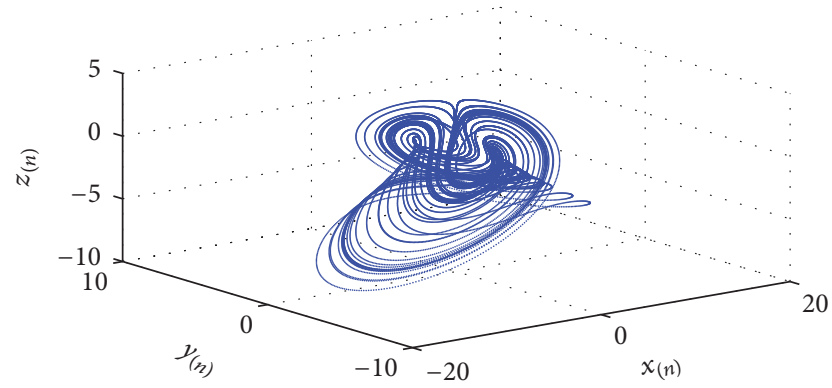

Figure 6: Chaotic attractor of DV-system (14) projected on $x_{(n)}$ versus $y_{(n)}$ versus $z_{(n)}$.

that the numerical results in simulation by using Matlab to represent the DV-system (14) and implementation by using Mikroc Pro for Pic 32 compiler are equivalents.

We use a novel method reported in $[37,38]$ in order to reproduce the DV of chaotic system (14) by using an PIC32 microcontroller and external DACs connected by the serial peripheral interface (SPI) protocol. The compact ES Mikromedia Plus for PIC32MX7 contains one 32bit PIC32MX795F512L microcontroller as central part. The Mikromedia Plus for PIC32MX7 ES allows development applications with multimedia contents, and it comes with several internal hardware-devices. We use the internal module TFTTSD (with one screen of 4.3 inches of $480 \times 272$ resolution) to represent, in real-time, the three phase planes of DV-system (12). TFT touch and LCD controller units are included into TFTTSD. Table 2 shows the hardware and the SPI modes description of the ES, and the schematic circuit diagram is shown in Figure 7. The evolution of discretized states $x_{(n)}, y_{(n)}$, and $z_{(n)}$ of DV-system (14) were reproduced by using the external DACs U1, U2, and U3, respectively.

System (14) describes $N=3$ dimension. To understand the simulation and implementation, the calculus of time was carried out in the algorithm of U1 to reproduce DV-system (14) on the ES. The time period $T_{\operatorname{Td}(N)}$ was considered as totaldecoding-time that the ES requires to process one iteration $n$. The maximum number of iterations $n$ that the ES generates in 1 second (i.p.s.) was calculated by frequency $f_{\operatorname{Td}(N)}$, that is, the reciprocal of $T_{\mathrm{Td}(N)}$; these terms are represented by

$$
T_{\operatorname{Td}(N)}=\frac{1}{f_{\operatorname{Td}(N)}}=t_{c}+t_{\operatorname{Tg}(N)},
$$

where the time complexity $t_{c}$ defines the time that the algorithm of U1 needs to reproduce one iteration $n$. The total-graphics-time $t_{\operatorname{Tg}(N)}$ is the time that U1 needs to enable the internal device TFTTSD and the DACs U2, U3, and U4 to reproduce in real-time one iteration $n$; we proposed the calculus of $T_{\mathrm{Td}(N)}$ considering DV-systems for $N$ dimensions, where $t_{\operatorname{Tg}(N)}$ was calculated externally of $t_{c}$. The total time required for each DAC is referred to as $t_{\mathrm{Tdac}(N)}$, and the time required for TFTTSD is referred to as $t_{\mathrm{tft}}$. The total-graphicstime is represented by

$$
\begin{aligned}
t_{\mathrm{Tg}(N)} & =t_{\mathrm{tft}}+\left(t_{\mathrm{dac}(j)}+t_{\mathrm{dac}(j+1)}+\cdots+t_{\mathrm{dac}(N)}\right) \\
& =t_{\mathrm{tft}}+\sum_{j=1}^{N} t_{\mathrm{Tdac}(j)}, \quad j=1,2, \ldots, N .
\end{aligned}
$$

In order to develop the equivalence between simulation and implementation on the ES, we defined the total quantity 

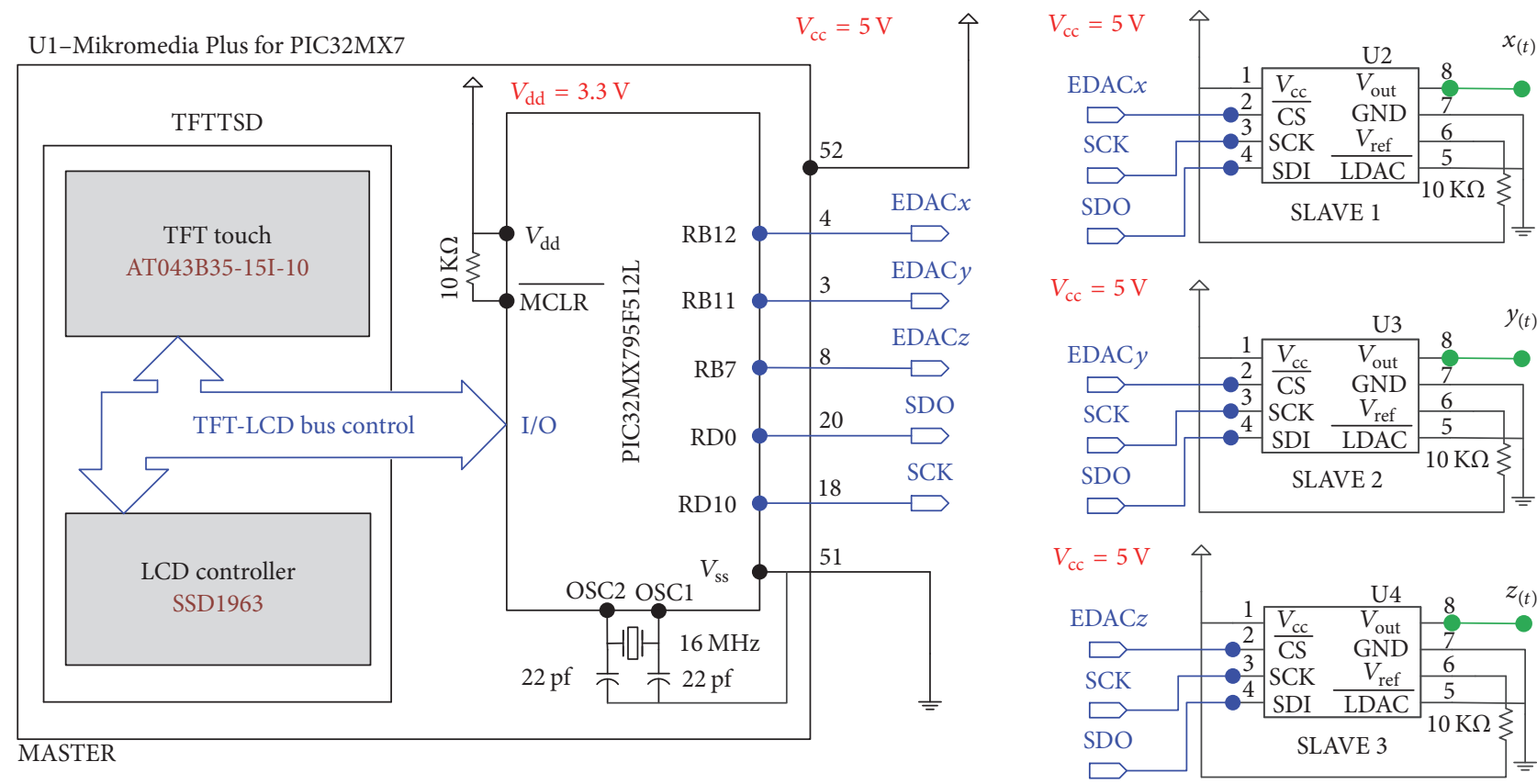

Figure 7: Schematic circuit design of ES for the implementation of DV-system (14).

TABLE 2: Main hardware description of ES.

\begin{tabular}{ll}
\hline Peripheral number & SPI mode, hardware description \\
\hline U1 & Master, TFTTSD Mikromedia Plus for PIC32MX7 \\
U2 & Slave 1, DAC MCP4921 shows $x_{(t)}$ \\
U3 & Slave 2, DAC MCP4921 shows $y_{(t)}$ \\
U4 & Slave 3, DAC MCP4921 shows $z_{(t)}$ \\
\hline
\end{tabular}

of iterations $Q_{T}$ as the maximum number of $n$ iterations generated in 1 second,

$$
Q_{T(N=3)}=\tau \frac{1}{T_{\operatorname{Td}(N)}}=\tau f_{\operatorname{Td}(N)} .
$$

The time for one specific number of iterations $n$ generated from the DV-system (14) is calculated by using the following expression:

$$
t_{n}=n\left[t_{c}+t_{\mathrm{Tg}(N)}\right]=n\left[t_{c}+t_{\mathrm{tft}}+t_{\mathrm{Tdac}(N)}\right] .
$$

Figure 8 and Table 3 show the implementation of system (14) to exemplify (15)-(18). Finally, we obtained $Q_{T}=9.17$ considering $\tau=0.01$; this means that in 1 second we obtained 9.17 time units. Figure $8(\mathrm{~d})$ shows $Q_{T}=91.7$ for $t=10 \mathrm{~s}$.

3.3. Degradation Study for DV of NCS. To prove the presence of chaos on the NCS (1), the LEs for discretized system (14) were calculated by using time series $[43,44]$. The result of Jacobean matrix for the discretized system (14) is

$$
J_{\mathrm{DV}}=\left(\begin{array}{ccc}
1-a \tau & -b \tau z_{(n)} & -b \tau y_{(n)} \\
-\tau & 1+c \tau & 0 \\
0 & -2 \tau y_{(n)} & 1-\tau
\end{array}\right),
$$

TABLE 3: Implementation results of DV-system (14) on the proposed ES.

\begin{tabular}{lc}
\hline Parameter & Value \\
\hline$\tau$ & 0.01 \\
$t_{\operatorname{Tg}(3)}$ & $27 \mu \mathrm{s}$ \\
$t_{\mathrm{tft}}$ & $24 \mu \mathrm{s}$ \\
$f_{\operatorname{Td}(3)}$ & $917 \mathrm{i} . \mathrm{p} . \mathrm{s}$. \\
$Q_{T(3)}$ & 9.17 \\
$t_{c}$ & $1063 \mu \mathrm{s}$ \\
$T_{\operatorname{Td}(3)}$ & $1090 \mu \mathrm{s}$ \\
$n$ & 917 \\
$t_{\operatorname{Tdac}(3)}$ & $3 \mu \mathrm{s}$ \\
$t_{(n=917)}$ & $0.99953 \mathrm{~s}$ \\
\hline
\end{tabular}

where the step size $\tau$ was modified as parameter to prove the chaotic behavior of the DV-system (14); $\tau$ is modified by using an increase of step size $\tau=0.001$ regarding 10000 time units, until the sign of the LEs changes and the discretized system (14) diverges. The LEs and fractal dimension of discretized system (14) are referred to as $L_{1}^{\prime}, L_{2}^{\prime}, L_{3}^{\prime}$ and $D_{\mathrm{KY}}^{\prime}$, respectively. Figure 9 and Table 4 show the result of chaos degradation corresponding to DV-system (14) for 2 cases. 


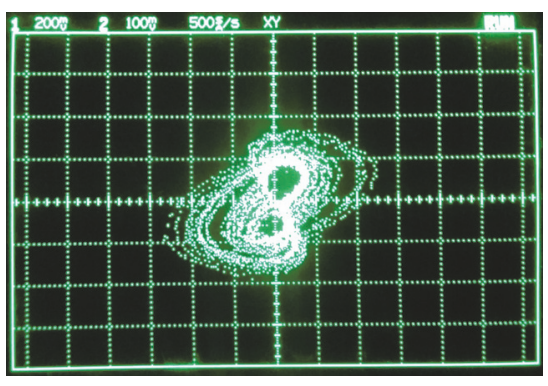

(a)

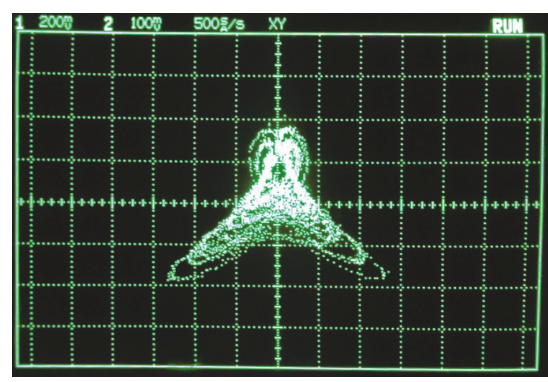

(b)

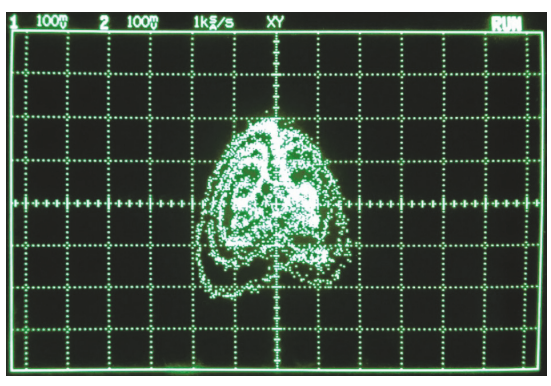

(c)

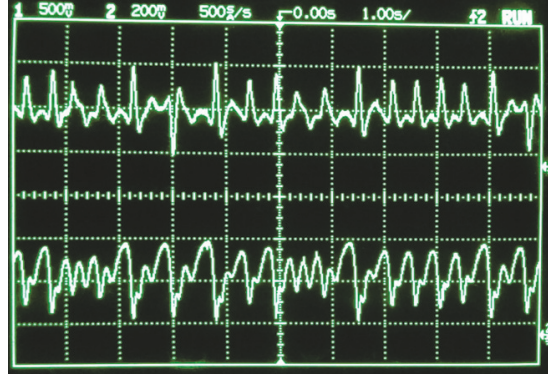

(d)

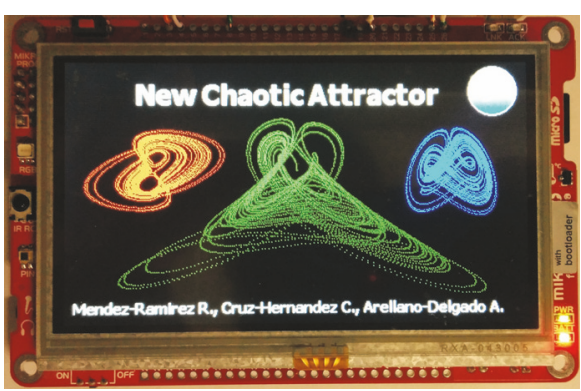

(e)

FIGURE 8: Implementation of DV-system (14) on proposed ES: (a) phase plane $x_{(n)}$ versus $y_{(n)}$, (b) phase plane $x_{(n)}$ versus $z_{(n)}$, (c) phase plane $y_{(n)}$ versus $z_{(n)}$, (d) time evolution of states $x_{(t)}$ and $z_{(t)}$ by using $\tau=0.01$ and $t=10 \mathrm{~s}$, and (e) representation of phase planes $x_{(n)}$ versus $y_{(n)}$, $x_{(n)}$ versus $z_{(n)}$, and $y_{(n)}$ versus $z_{(n)}$ on TFTTSD.

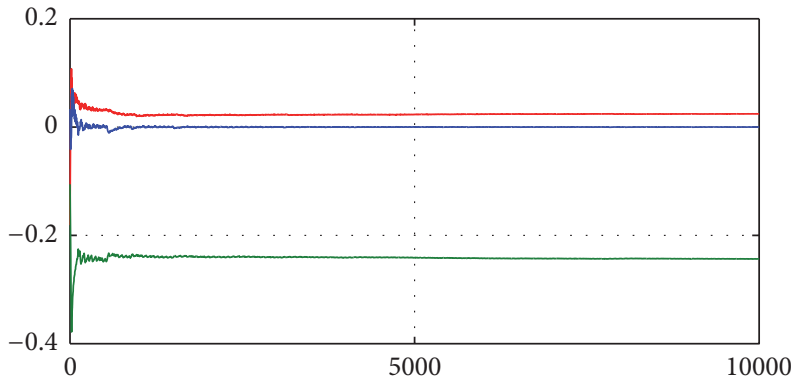

$-L_{1}^{\prime}$
$-L_{2}^{\prime}$
$-L_{3}^{\prime}$

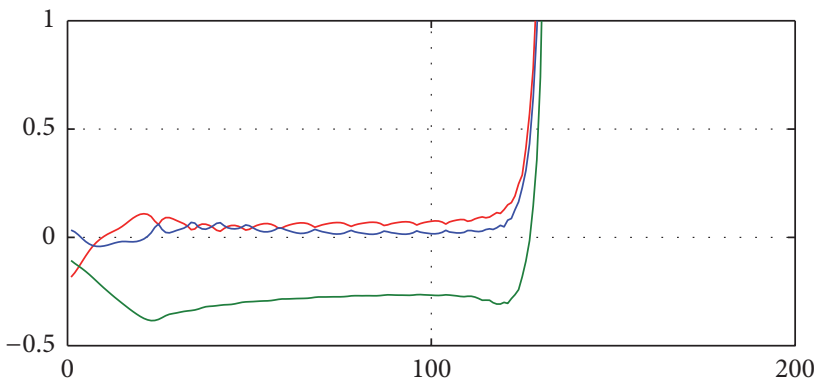

$\begin{aligned}- & L_{1}^{\prime} \\ - & L_{2}^{\prime} \\ - & L_{3}^{\prime}\end{aligned}$

(a)

(b)

FIGURE 9: LEs of discretized system (14) for (a) $\tau=0.085$ and (b) $\tau=0.086$.

TABLE 4: Analysis of chaos degradation for DV-system (14) by using LEs.

\begin{tabular}{lccc}
\hline Case & $\tau$ & LEs & Result \\
\hline & & $L_{1}^{\prime}=+0.050763$ & \\
1 & $(0,0.085]$ & $L_{2}^{\prime}=-0.000064588$ & Chaotic behavior shown in Figure 9(a) \\
& $L_{3}^{\prime}=-0.24347$ & \\
\hline & $D_{\mathrm{KY}}^{\prime}=2.0996$ & No displayed chaos, see Figure 9(b) \\
& $L_{1}^{\prime}=$ no valid & $L_{2}^{\prime}=$ no valid & \\
\hline
\end{tabular}


TABLE 5: Comparison of the proposed NCS with some chaotic systems reported in the literature.

\begin{tabular}{lccccccc}
\hline Chaotic system & Parameters & Critical parameters & Nonlinearities & Step size $\tau$ & Total time $\mu$ s & Iterations per second & Time units $Q_{T}$ \\
\hline Lorenz & $\sigma, b, c$ & $\sigma$ & 2 & $\leq 0.024$ & 1090 & 917 & 22 \\
Rössler & $a, b, c$ & $c$ & 1 & $\leq 0.005$ & 1073 & 932 & 9.7 \\
Chen & $a, b, c$ & $a$ & 2 & $\leq 0.002$ & 1090 & 917 & 912 \\
Liu and Chen & $a, b, c, d_{1}, d_{2}, d_{3}$ & $c$ & 3 & $\leq 0.002$ & 1096 & 1.8 & 917 \\
Proposed NCS & $a, b, c, d$ & $b, d$ & 2 & $\leq 0.085$ & 1090 & 78 \\
\hline
\end{tabular}

For case 1, the discretized system (14) conserves the chaotic behavior. This result was compared with the LEs calculated for CV of system (1) where the numerical results of $L_{1}$ and $D_{\mathrm{KY}}$ were similar with respect to $L_{1}^{\prime}$ and $D_{\mathrm{KY}}^{\prime}$. The maximum step size $\tau=0.085$ was found. For case 2, the step size was increased until obtaining $\tau=0.086$, whereby LEs can not be calculated in the DV-system (14). For values of $\tau \geq 0.086$, the discretized system (14) diverges and the state trajectories $x_{(n)}, y_{(n)}$, and $z_{(n)}$ can not display chaos.

3.4. Comparison of the Proposed NCS with Some Chaotic Systems. In order to compare the performance of the NCS (14) in DV, we studied the chaotic degradation of four 3D Lorenz, Rössler, Chen, and Liu and Chen classical chaotic systems where their DVs were obtained by using the same Euler discretization (13), and the LEs were calculated by using the same method as in $[43,44]$. Table 5 shows the results of the step sizes $\tau$ intervals of the five Lorenz, Rössler, Chen, and Liu and Chen CSs using the Euler numerical algorithm (13) where the chaotic behavior is conserved in these chaotic systems $[16,17,22,23]$. According to Table 5, the proposed NCS in DV (14) presents a higher step size with respect to the other four 3D Lorenz, Rössler, Chen, and Liu and Chen chaotic systems in DV. This means that, for implementation, the NCS in DV has more compacts dynamics to digital implementations; then the NCS in DV is a good alternative using ESs where the main part has less processing capacity, for example, 8-bit microcontrollers family. The novelty of the proposed chaotic system is the combination of the different characteristics that it presents, which results in a high ease of implementation for its use in different applications as previously mentioned.

\section{Digital Implementation Process}

In this section, we present the flow chart and the description of the electronical/digital implementation process that contains the proposed programming algorithm for the implementation of the NSC in DV (14). In addition, we present some aspects of implementation robustness from the point of view of software and hardware, a study regarding the robustness of the critical parameters, and comparative advantages of the implementation for the NSC in DV (14).

4.1. Flow Chart Digital Implementation. In Figure 10, we illustrate the flow chart of the general electronical/digital implementation process. The description of each step is described below.
Step 1. Set initial calibration of the TFTTSD U1. The TFTTSD is initialized and an internal program that allows calibrating the internal TFT touch and LCD controllers of the TFTTSD is executed; the four edges of the TFT screen are used.

Step 2. Set graphic environment, variables, and parameters of NCS in DV (14) on PIC32MX795F512L microcontroller. The floating point and decimal-base constants to be used in the programming algorithm of the NSC in DV (14) are defined.

Step 3. Initialization of ports and SPI protocol: the SPI protocol of the main PIC32MX795F512L microcontroller is configured in master mode and the SPI of the external DACs $\mathrm{U} 1, \mathrm{U} 2$, and $\mathrm{U} 3$ are configured in slave mode.

Step 4. Set the critical parameters, initial conditions $x_{0}, y_{0}, z_{0}$, and step size $\tau$ for NCS in DV (14). The critical parameters $a=2, b=2, c=0.5$, and $d=4$, initial conditions $x_{0}=y_{0}=z_{0}=1$, and step size $\tau=0.004$ corresponding to the initial iteration of the NCS in DV (14) are defined.

Step 5. Definition of the NCS in DV (14) using Euler's numerical algorithm. The discretized NCS is defined by the Euler's numerical algorithm. Delay time in this stage is $14 \mu \mathrm{s}$.

Step 6. Storing the current values of state variables $x_{(n)}, y_{(n)}$, and $z_{(n)}$ : this value corresponds to the next iteration of the NCS in DV (14). Delay time in this stage is $0.2 \mu \mathrm{s}$.

Step 7. Rescaling the state variables $x_{(n)}, y_{(n)}$, and $z_{(n)}$ in positive scale: Representation of the state variables $x_{(n)}, y_{(n)}$, and $z_{(n)}$ is rescaled, since the numerical representation in the TFTTSD and the DACs is positive. Delay time in this stage is $1033.7 \mu \mathrm{s}$.

Step 8. Rescaling the values of state variables $x_{(n)}, y_{(n)}$, and $z_{(n)}$ for the TFTTSD in $480 \times 272$ resolution: to display images in the TFTTSD, Visual TFT software is used to design a template that displays graphics and text. In our case, the evolution of the phase planes $x_{(n)}$ versus $y_{(n)}, x_{(n)}$ versus $z_{(n)}$, and $y_{(n)}$ versus $z_{(n)}$ is shown in real-time. In addition, the names of the authors are shown. Delay time in this stage is $7.5 \mu \mathrm{s}$.

Step 9. Write the TFTTSD using the TFT library to draw a dot at certain coordinates for each phase plane: $x_{(n)}$ versus $y_{(n)}$ in red color, $x_{(n)}$ versus $z_{(n)}$ in green color, and $y_{(n)}$ versus $z_{(n)}$ in blue color. Once the values are rescaled within the TFTTSD resolution, the "TFT_Dot" library is used to display a point 


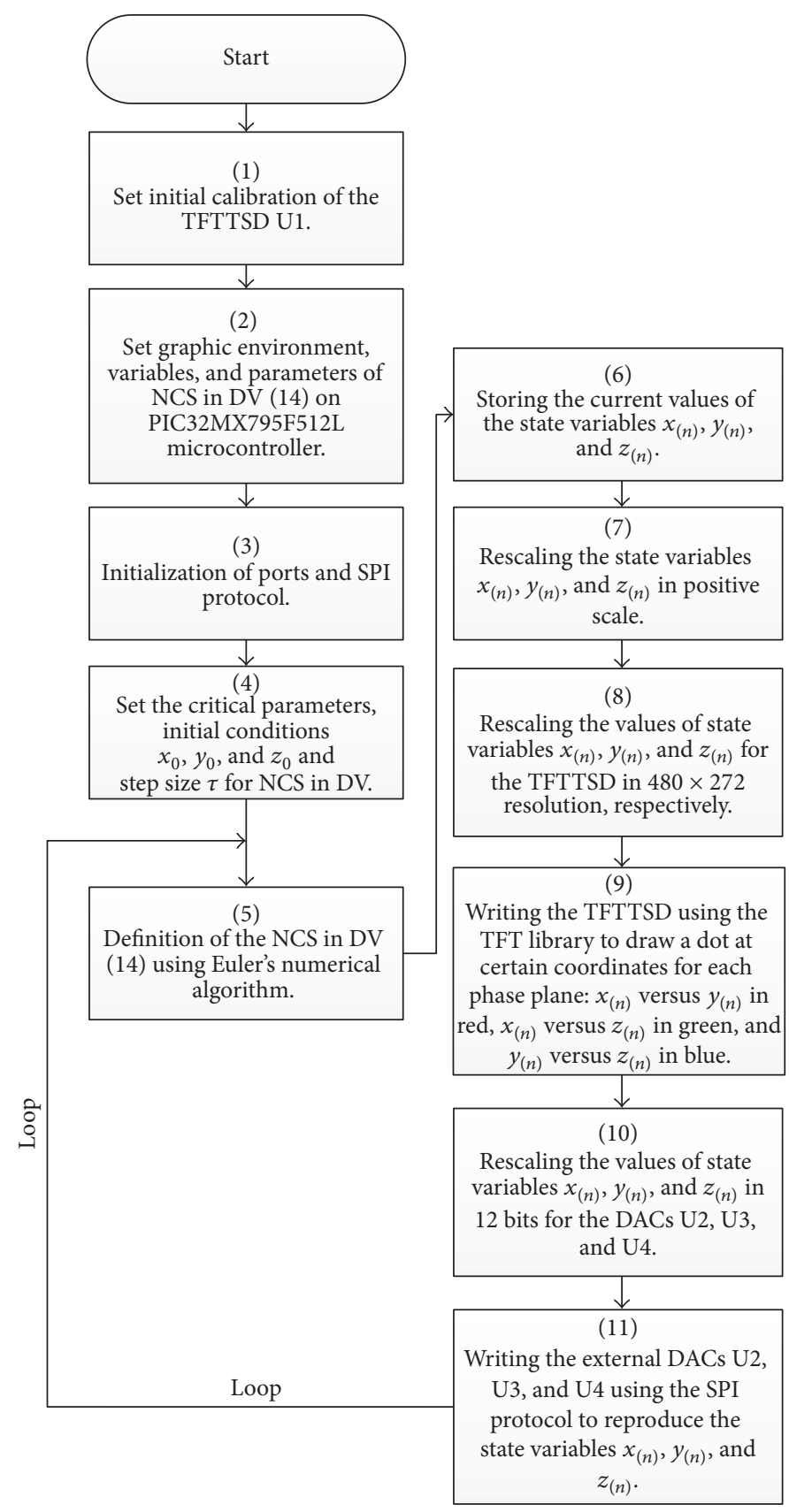

FIGURE 10: Flow chart of the general electronical/digital implementation process.

with a different color according to the coordinates indicated by the phase planes of each state variable. Delay time in this stage is $24 \mu \mathrm{s}$.

Step 10. Rescaling the values of state variables $x_{(n)}, y_{(n)}$, and $z_{(n)}$ in 12 bits for the DACs U2, U3, and U4: for the implementation of the state variables $x_{(n)}, y_{(n)}$, and $z_{(n)}$, a positive scale is used with a maximum resolution interval of 12 bits, from 0 to 4095 which is the working range of the DACs and the SPI protocol, resulting in $16 \mathrm{Mbps}$. Delay time in this stage is $7.6 \mu \mathrm{s}$.
Step 11. Write the external DACs U2, U3, and U4 using the SPI protocol to reproduce the state variables $x_{(n)}, y_{(n)}$, and $z_{(n)}$. The microcontroller PIC32MX795F512L configured in master mode is used to enable the select chip and write the DACs U1, U2, and U3 (configured in slave mode), where the state variables $x_{(n)}, y_{(n)}$, and $z_{(n)}$ are reproduced simultaneously. Delay time in this stage is $3 \mu \mathrm{s}$.

Finally, a loop from Steps $11-5$ is performed where the parameter values of the NSC in DV (14) and $\tau$ were previously defined according to Table 4. 


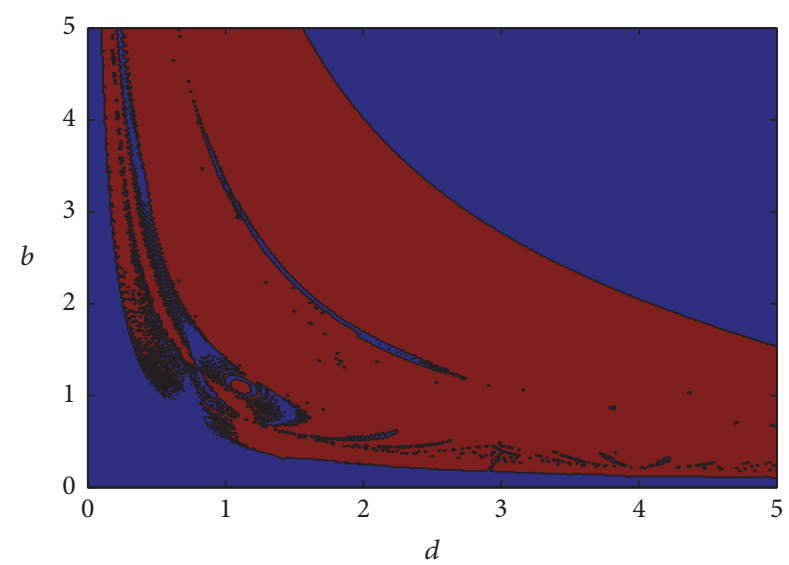

FIGURE 11: Robustness diagram to determine chaos existence for $b$ versus $d$ at intervals of 0.01 and with $\tau=0.085$ : chaos (red), no chaos (blue).

4.2. Robustness in the Implementation of the NCS Digital Version. According to [47], software robustness is the ability of a product to stay in service and function correctly even with the occurrence of errors that are attributable to hardware, software, or even external influences. The implemented software in the TFTTSD is designed from graphical interface tools using the Visual TFT in conjunction with programming code designed in $\mathrm{C}$ language that is stored in the PIC32MX795F512L microcontroller flash memory. The accuracy of the programming algorithm calculations depends on the IEEE-754 AN575 standardization of the PIC32MX795F512L microcontroller [46]. With regard to hardware, the TFTTSD has two possible forms of energization: the first is through the USB port connected to a laptop or desktop PC; the second is via an external lithium battery. The possibility that the TFTTSD can be energized through an external battery makes it portable, which allows the autonomy of the equipment.

On the other hand, in order to show the robustness of chaos presence in the discretized system (14), a robustness diagram based on the variation of critical parameters $b$ and $d$ was carried out. In this diagram it is possible to determine the regions in which the existence of chaos is guaranteed, considering $\tau=0.085$. Figure 11 shows the regions of chaos existence for $b$ versus $d$ (intervals of 0.01 are used for both parameters $b$ and $d$ ) where each point in the graph represents the maximum Lyapunov exponent $\left(L_{\max }^{\prime}\right)$. If we have $L_{\max }^{\prime}>$ 0 , that is, if the dynamics are chaotic, the red color is used; otherwise, the blue color is used. From the robustness diagram in Figure 11 it can be seen that the chaotic dynamics are preserved for wide intervals of the parameter values $b$ and $d$.

Furthermore, it is easy to note that if a value of $\tau$ less than 0.085 is considered, then the chaos regions increase. Taking into account the fact that the preservation of chaos in the discretized version of the NCS proposed is robust for the parameters $b$ and $d$, considering the software and hardware characteristics of the proposed ES, and the benefits of digital systems, as the elimination of the typical wear of the analog systems, it is stated that the electronical/digital implementation presented in this work is robust.
On the other hand, to the best of our knowledge the electronical implementation in a portable TFTTSD device of DV of chaotic systems, for the reproduction of their nonlinear dynamics in real-time, is new. By having a graphical interface and given certain potential applications in the engineering field, such as biometric systems, telemedicine, cryptography, and secure communications, the proposed digital implementation makes the interaction between the device and the end user very friendly. One of the most relevant advantages of the NSC in DV (14) is the increase in step size compared with other chaotic systems, which allows implementation in slower microcontrollers, for example, in 8-bit low-end microcontrollers; microchip PIC microcontrollers, Motorola M68HC05 microcontrollers, AVR microcontrollers, ATmega328, and 8051 from the manufacturer Atmel. In the same way, there are alternative families of 16bit mid-range microcontrollers to implement the NSC in DV (14), such as the dsPIC family of manufacturer microchip, MSP430 of Texas Instruments.

Finally, we can find the high-end microcontrollers, which are those used in the implementation presented in this work. A microchip PIC32 microcontroller was used which shows great benefits in the use of TFTTSD; along with this microcontroller there are other alternatives such as the STM32 microcontrollers of the manufacturer STMicroelectronics or the FT900 microcontrollers of the manufacturer Future Technology Devices International Limited.

\section{Conclusions}

We have proposed a new chaotic system (NCS), which generates chaotic dynamics varying two parameters.

Analytical and numerical studies to confirm the chaos generation for continuous and discretized version (DV) were presented. Also, a degradation analysis on the discretized version of the NCS was carried out to find the maximum step size. The results showed that the NCS is flexible and robust which allows obtaining different chaotic behaviors.

In addition, the NCS was implemented electronically for continuous version with operational amplifiers and for DV we used a novel embedded system that shows dynamical behaviors in real-time.

As future work, the authors will concentrate on carrying out a complete analysis of the proposed chaotic system, providing rigorous mathematical proofs, to estimate the ultimate bound and positively invariant set, as is reported in the current literature [11,48-50], and, in addition, to apply these analytical results to synchronize the proposed chaotic system via approach reported in [51].

\section{Conflicts of Interest}

The authors declare that there are no conflicts of interest regarding the publication of this paper.

\section{Acknowledgments}

This work is supported by the CONACYT, México, under Research Grant 166654. 


\section{References}

[1] J. Guckenheimer and P. Holmes, Nonlinear Oscillations, Dynamical Systems, and Bifurcation of Vector Fields, Springer, New York, NY, USA, 1982.

[2] S. Wiggins, Introduction to Applied Nonlinear Dynamical Systems and Chaos, Springer, Berlin, Germany, 1990.

[3] S. H. Strogatz, Nonlinear dynamics and chaos: with applications to physics, biology, chemistry, and engineering, Perseus Books, Massachusetts, USA, 1994.

[4] W. Xingyuan and L. Chao, "Researches on chaos phenomenon of EEG dynamics model," Applied Mathematics and Computation, vol. 183, no. 1, pp. 30-41, 2006.

[5] K.-Z. Li, M.-C. Zhao, and X.-C. Fu, "Projective synchronization of driving-response systems and its application to secure communication," IEEE Transactions on Circuits and Systems. I. Regular Papers, vol. 56, no. 10, pp. 2280-2291, 2009.

[6] H. O. Wang, E. H. Abed, and A. M. A. Hamdan, "Bifurcations, chaos, and crises in voltage collapse of a model power system," IEEE Transactions on Circuits and Systems I: Fundamental Theory and Applications, vol. 41, no. 4, pp. 294-302, 1994.

[7] F.-Y. Lin and J.-M. Liu, "Chaotic radar using nonlinear laser dynamics," IEEE Journal of Quantum Electronics, vol. 40, no. 6, pp. 815-820, 2004.

[8] R. V. Donner, J. Heitzig, J. F. Donges, Y. Zou, N. Marwan, and J. Kurths, "The geometry of chaotic dynamics-a complex network perspective," The European Physical Journal B. Condensed Matter and Complex Systems, vol. 84, no. 4, pp. 653-672, 2011.

[9] A. Arellano-Delgado, R. M. López-Gutiérrez, C. CruzHernández, C. Posadas-Castillo, L. Cardoza-Avendaño, and H. Serrano-Guerrero, "Experimental network synchronization via plastic optical fiber," Optical Fiber Technology, vol. 19, no. 2, pp. 93-98, 2013.

[10] M. A. Murillo-Escobar, C. Cruz-Hernández, F. Abundiz-Pérez, R. M. López-Gutiérrez, and O. R. Acosta Del Campo, "A RGB image encryption algorithm based on total plain image characteristics and chaos," Signal Processing, vol. 109, pp. 109131, 2015.

[11] H. Saberi Nik, S. Effati, and J. Saberi-Nadjafi, "Ultimate bound sets of a hyperchaotic system and its application in chaos synchronization," Complexity, vol. 20, no. 4, pp. 30-44, 2015.

[12] M. A. Murillo-Escobar, C. Cruz-Hernández, F. Abundiz-Pérez, and R. M. López-Gutiérrez, "A robust embedded biometric authentication system based on fingerprint and chaotic encryption," Expert Systems with Applications, vol. 42, no. 21, pp. 81988211, 2015.

[13] M. A. Murillo-Escobar, L. Cardoza-Avendaño, R. M. LópezGutiérrez, and C. Cruz-Hernández, "A Double Chaotic Layer Encryption Algorithm for Clinical Signals in Telemedicine," Journal of Medical Systems, vol. 41, p. 59, 2017.

[14] Y. Yan, "Synchronization for a class of uncertain fractional order chaotic systems with unknown parameters using a robust adaptive sliding mode controller," Hindawi Publishing Corporation Mathematical Problems in Engineering, vol. 2016, Article ID 7404652, 7 pages, 2016.

[15] J. Zhang, D. Hou, and H. Ren, "Image encryption algorithm based on dynamic DNA coding and Chen's hyperchaotic system," Mathematical Problems in Engineering, vol. 2016, Article ID 6408741, 11 pages, 2016.

[16] E. Lorenz, "Deterministic nonperiodic flow," Journal of the Atmospheric Sciences, vol. 20, pp. 130-141, 1963.
[17] O. E. Rössler, "An equation for continuous chaos," Physics Letters A, vol. 57, no. 5, pp. 397-398, 1976.

[18] J. Lü and G. Chen, "A new chaotic attractor coined," International Journal of Bifurcation and Chaos in Applied Sciences and Engineering, vol. 12, no. 3, pp. 659-661, 2002.

[19] L. O. Chua, "The Double Scroll Family," IEEE Transactions on Circuits and Systems, vol. 33, no. 11, pp. 1072-1118, 1986.

[20] C. Liu, T. Liu, L. Liu, and K. Liu, "A new chaotic attractor," Chaos, Solitons and Fractals, vol. 22, no. 5, pp. 1031-1038, 2004.

[21] J. C. Sprott, "Some simple chaotic flows," Physical Review E. Statistical, Nonlinear, and Soft Matter Physics, vol. 50, no. 2, part A, pp. R647-R650, 1994.

[22] G. Chen and T. Ueta, "Yet another chaotic attractor," International Journal of Bifurcation and Chaos in Applied Sciences and Engineering, vol. 9, no. 7, pp. 1465-1466, 1999.

[23] W. B. Liu and G. Chen, "A new chaotic system and its generation," International Journal of Bifurcation and Chaos, vol. 12, pp. 261-267, 2002.

[24] J. C. Sprott, Elegant Chaos Algebraically Simple Chaotic Flows, World Scientific, Singapore, 2010.

[25] C. Gissinger, "A new deterministic model for chaotic reversals," European Physical Journal B, vol. 85, no. 137, 2012.

[26] C. Li and J. C. Sprott, "Multistability in a butterfly flow," International Journal of Bifurcation and Chaos in Applied Sciences and Engineering, vol. 23, no. 12, pp. 1350199-1350209, 2013.

[27] W. T. Verkley and C. A. Severijns, "The maximum entropy principle applied to a dynamical system proposed by Lorenz," The European Physical Journal B. Condensed Matter and Complex Systems, vol. 87, no. 7, 2014.

[28] J. Wu, L. Wang, G. Chen, and S. Duan, "A memristive chaotic system with heart-shaped attractors and its implementation, Chaos," Solitons Fractals, vol. 92, pp. 20-29, 2016.

[29] A. L'Her, P. Amil, N. Rubido, A. C. Marti, and C. Cabeza, "Electronically-implemented coupled logistic maps," The European Physical Journal B. Condensed Matter and Complex Systems, vol. 89, no. 81, 2016.

[30] L. J. Ontañón-García and E. Campos-Cantón, "Preservation of a two-wing Lorenz-like attractor with stable equilibria," Journal of the Franklin Institute. Engineering and Applied Mathematics, vol. 350, no. 10, pp. 2867-2880, 2013.

[31] A. T. Azar, C. Volos, N. . Gerodimos et al., "A novel chaotic system without equilibrium: dynamics, synchronization, and circuit realization," Hindawi Publishing Corporation Complexity, vol. 2017, Article ID 7871467, 11 pages, 2017.

[32] X. Wang, V.-T. Pham, and C. Volos, "Dynamics, circuit design, and synchronization of a new chaotic system with closed curve equilibrium," Hindawi Publishing Corporation Complexity, vol. 2017, Article ID 7138971, 9 pages, 2017.

[33] M. P. Mareca and B. Bordel, "Improving the complexity of the Lorenz dynamics," Hindawi Publishing Corporation Complexity, vol. 2017, Article ID 3204073, 16 pages, 2017.

[34] C. Cruz-Hernández, D. López-Mancilla, V. García-Gradilla, H. Serrano-Guerrero, and R. Núñez-Pérez, "Experimental realization of binary signals transmission using chaos," in Proceedings of the 1st International Conference on Communications, Circuits and Systems (ICCCAS '02), pp. 146-149, July 2002.

[35] Q. Wang, S. Yu, C. Li et al., "Theoretical design and FPGA-based implementation of higher-dimensional digital chaotic systems," IEEE Transactions on Circuits and Systems. I. Regular Papers, vol. 63, no. 3, pp. 401-412, 2016. 
[36] B. Cai, G. Wang, and F. Yuan, "Pseudo random sequence generation from a new chaotic system," in Proceedings of the 16th IEEE International Conference on Communication Technology (ICCT '15), pp. 863-867, October 2015.

[37] R. Méndez-Ramírez, A. Arellano-Delgado, C. Cruz-Hernández, F. Abundiz-Pérez, and R. Martínez-Clark, "Chaotic Digital Cryptosystem by using SPI Protocol and its dsPICs Implementation," Frontiers of Information Technology Electronic Engineering.

[38] R. Mendez-Ramirez, A. Arellano-Delgado, C. Cruz-Hernandez, and R. M. Lopez-Gutierrez, "Degradation analysis of generalized Chua's circuit generator of multi-scroll chaotic attractors and its implementation on PIC32," in Proceedings of the Future Technologies Conference (FTC), pp. 1034-1039, San Francisco, CA, USA, December 2016.

[39] L. Acho, "A discrete-time chaotic oscillator based on the logistic map: a secure communication scheme and a simple experiment using Arduino," Journal of the Franklin Institute. Engineering and Applied Mathematics, vol. 352, no. 8, pp. 3113-3121, 2015.

[40] Q. Yang and G. Chen, "A chaotic system with one saddle and two stable node-foci," International Journal of Bifurcation and Chaos in Applied Sciences and Engineering, vol. 18, no. 5, pp. 1393-1414, 2008.

[41] H. S. Nik and M. Golchaman, "Chaos Control of a Bounded 4D Chaotic System," Neural Comput Applic, vol. 25, no. 3, pp. 683692, 2014.

[42] M. Suneel, "Electronic circuit realization of the logistic map," Sadhana, vol. 31, no. 1, pp. 69-78, 2006.

[43] A. Wolf, J. B. Swift, H. L. Swinney, and J. A. Vastano, "Determining Lyapunov exponents from a time series," Physica D. Nonlinear Phenomena, vol. 16, no. 3, pp. 285-317, 1985.

[44] K. Briggs, "An improved method for estimating Liapunov exponents of chaotic time series," Physics Letters. A, vol. 151, no. 1-2, pp. 27-32, 1990.

[45] W. Y. Yang, W. Cao, T.-S. Chung, and J. Morris, Applied numerical methods using Matlab, John Wiley and Sons Inc., 2005.

[46] Microchip Technology Inc., "AN575. IEEE-754 Compliant Floating Point Routines," in DS00575B, pp. 1-155, 1997.

[47] S. Fraser, D. Campara, C. Chilley et al., "Fostering software robustness in an increasingly hostile world," in Proceedings of the Companion to the 20th annual ACM SIGPLAN conference, p. 378, San Diego, CA, USA, October 2005.

[48] G. A. Leonov, A. I. Bunin, and N. Koksch, "Attraktorlokalisierung des Lorenz-Systems," Zeitschrift für Angewandte Mathematik und Mechanik, vol. 67, no. 12, pp. 649-656, 1987.

[49] A. Y. Pogromsky, G. Santoboni, and H. Nijmeijer, "An ultimate bound on the trajectories of the Lorenz system and its applications," Nonlinearity, vol. 16, no. 5, pp. 1597-1605, 2003.

[50] D. Li, J. Lu, X. Wu, and G. Chen, "Estimating the bounds for the Lorenz family of chaotic systems, Chaos," Solitons Fractals, vol. 23, pp. 529-534, 2005.

[51] H. Sira-Ramírez and C. Cruz-Hernández, "Synchronization of chaotic systems: a generalized Hamiltonian systems approach," International Journal of Bifurcation and Chaos in Applied Sciences and Engineering, vol. 11, no. 5, pp. 1381-1395, 2001. 


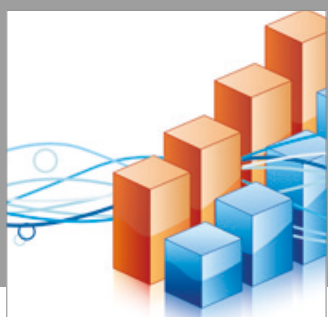

Advances in

Operations Research

vatersals

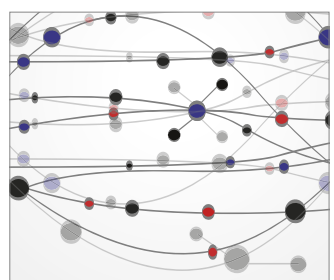

\section{The Scientific} World Journal
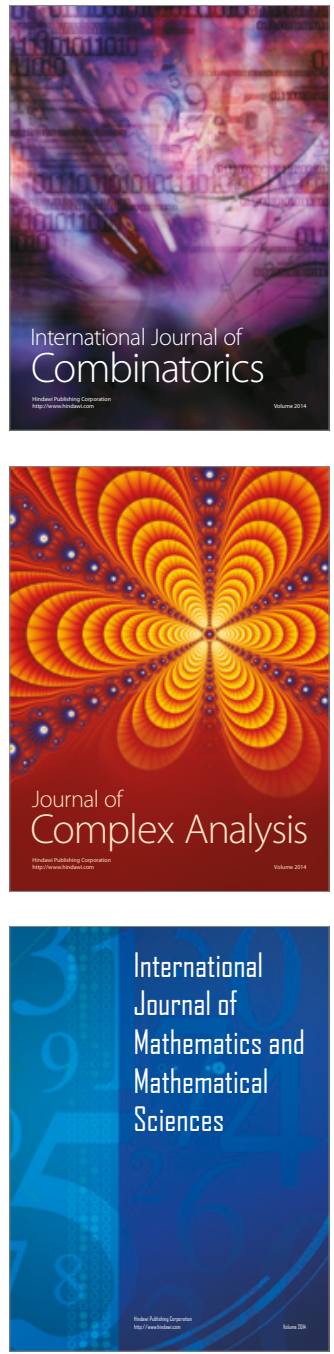
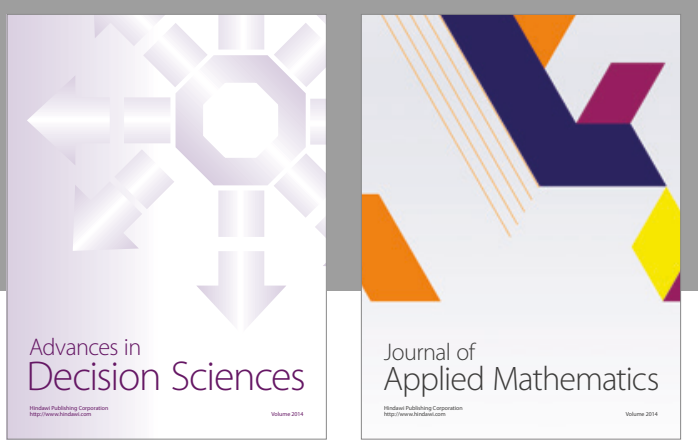

Algebra

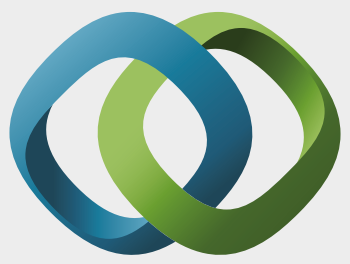

\section{Hindawi}

Submit your manuscripts at

https://www.hindawi.com
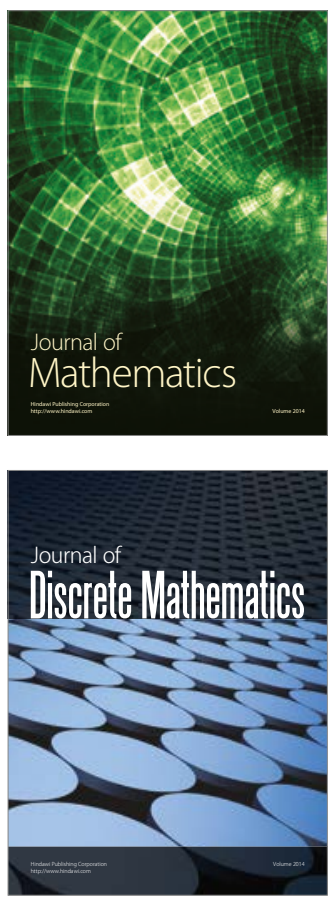

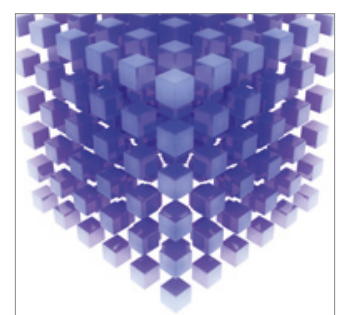

Mathematical Problems in Engineering
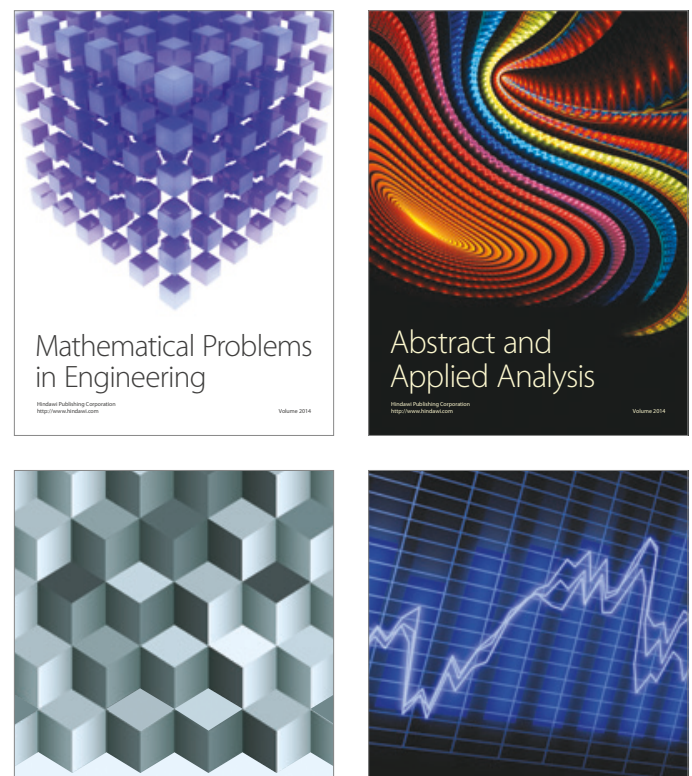

Journal of

Function Spaces

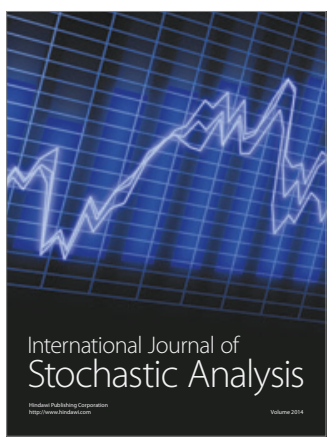

Probability and Statistics
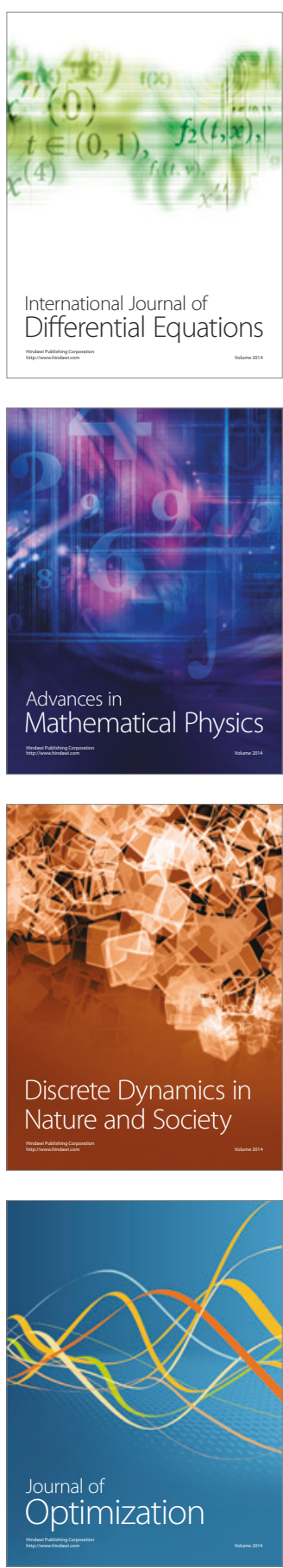